\title{
J-Spectral Factorization
}

\author{
M. Šebek * \\ Institute of Information Theory and Automation \\ Czechoslovak Academy of Sciences \\ 18208 Prague 8, Czechoslovakia
}

\author{
H. Kwakernaak \\ Faculty of Applied Mathematics \\ University of Twente \\ 7500 AE Enschede, the Netherlands
}

\begin{abstract}
The problem of $J$-spectral factorization of polynomial matrices is studied. Existence and uniqueness are discussed and the differences from the standard case are pointed out. Two first algorithms are proposed for a numerical solution.
\end{abstract}

\section{Introduction}

The problem of spectral factorization of a polynomial matrix appears to be important for practical use in many areas of science and engineering. Namely, the spectral factorization of a polynomial matrix which is positive definite on the imaginary axis, called here the standard spectral factorization, has grown into one of the basic tools of modern control theory for its service to linear quadratic $\left(\mathcal{H}_{2}\right.$-)optimal control $[10,6]$. In addition, a new interesting area of application for matrix spectral factorization has arisen recently in $\mathcal{H}_{\infty}$-optimal control. For this purpose, the task is to compute the spectral factor of a polynomial matrix indefinite on the imaginary axis (the so called $J$-spectral factorization) [7].

Several numerical algorithms to solve the standard factorization have been developed in the last three decades. They are based on computation of zeros and symmetric factor extraction, on Cholesky lactorization of a block Toeplitz matrix constructed from matrix coefficients, on Newton-Raphson method and on the solution of algebraic Riccati equation. Among them, chiefly the last two are now very popular. None of the above methods can be implemented directly to solve the $J$-spectral factorization. In many cases. in fact, the problems connected with their application seem to be principal ones [9].

It is the aim of this paper to look at the problem of $J$-spectral factorization a little more deeply, to point out the differences from the standard case and, namely, to present two first algorithms for a numerical solution.

The first algorithm incorporates the computation of zeros and a generalized version of the symmetric factor extraction. It features simplicity as well as a nice indication of certain internal singularity which, in fact, reflects the $\mathcal{H}_{\infty}$-optimality and can be employed to compute directly the reduced optimum compensator [7].

The second one is a new procedure inspired partially by a mathematical work of Jakubovic [5]. It is based on diagonalization, scalar spectral factorization and the backward construction of the matrix factor. It is more complex but it exhibits a little better numerical properties as it avoids the need to compute the zeros.

*The work was done while the first author was with the Systems and Control (iroup and Mechatronics Research Center of the University of Twente.
It handles matrices of any signature (including singular ones) and requires no particular form of them to start. It seems to be numerically attractive even for the standard (positive definite) case since. in contrast to all other iterative methods, the iteration is performed just on scalar polynomials so that all typical numerical troubles (appearing with multiple zeros on the stability boundary) are beaten down.

\section{Existence and Uniqueness}

Let us first recall that an adjoint $A^{*}(s)$ of a real polynomial matrix $A(\mathrm{~s})$ is defined by $A^{*}(\mathrm{~s})=A^{T}(-\mathrm{s})$ and the matrix $A(\mathrm{~s})$ is called paru-llcrmition if $A^{*}(\mathrm{~s})=A(\mathrm{~s})$. A general real spectral factorization can be then defined as follows:

Definition 1 Let be given a para-Hermitian ${ }^{1}$ real polynomial matrix $A(\mathrm{~s})$. Any representation

$$
A(\mathrm{~s})=C^{*}(\mathrm{~s}) K C(\mathrm{~s})
$$

where $C(\mathrm{~s})$ is a real non-singular square polynomial matrix and $K$ is a symmetric real constant matrix $\left(K^{T}=K\right)$ is labelled (right $^{2}$ ) spectral factorization of $A(\mathbf{s})$ and any $C(\mathrm{~s})$ satisfying (1) is collerl (right) spectral factor of $A(\mathrm{~s})$. Moreover, the $C(\mathrm{~s})$ is a Hurwitz spectral factor of $A(s)$ whenever $C(s)$ is a Hurwitz ${ }^{3}$ matrix in addition.

As applications are limited solely to the Hurwitz spectral factors, just those will be considered throughout the paper while the adjective "Hurwitz" will be dropped for brevity.

Remark 1 Once the spectral factor exists, the real matrix $K$ can always found in the (block) diagonal form

$$
K=\operatorname{diag}\left\{I_{p},-I_{n}, 0_{o \times o}\right\},
$$

which is the so called signature matrix ${ }^{4}$. Without any lost in generality, we shall consider only such $K$ 's throughout the paper. The clefinition above covers a variety of matrix factorization problenss. In particular, if $A(\mathrm{~s})$ is positive (non-negative) definite on the imaginary axis, we refer to the standard case and always $K=I$ results. On the other hand, if $A(s)$ is indefinite on the imaginary axis, we speak of $J$-spectral factorization and, as usually, we write $J\left(=\operatorname{diag}\left\{I_{p},-I_{n}\right\}\right)$ instead of $K$.

\footnotetext{
'No symmetric factorization (1) evidently exists unless $A(s)$ is paraHermitian. Hence it is 110 loss in generality to consider just such matrices

"The dual (left or co-) factorization $A(s)=C(s) K C^{*}(s)$ can be found simply by applying (1) to $A^{T}(s)$ and taking the transpose of the result.

${ }^{3}$ In the context of continuous-time systems, which is considered here, a polynomial matrix is called Hurwitz if it has no open right half-plain zeros.

${ }^{4}$ Besides. the signature of a real symmetric matrix $K$ is a triple $(p, n, o)$ consisting of the number of positive. negative and zero eigenvalues of $K$, respectively.
} 
The condition for the spectral factorization to exist is as follows:

Theorem $1 \mathrm{~A} n(n \times n)$ polynomial matrix $A(\mathrm{~s})$ possesses a spectral factorization (1) if for every nonzero invariant polynomial $\mathrm{i}_{l}(A)(\mathbf{s})$ of $A(\mathbf{s}), i=1, \ldots, n$, holds that if it possesses zeros on $\operatorname{Re} s=0$. then they must be of even multiplicity.

Proor:

See $[5.9]$.

To put it in another words, the sufficient condition is violated only if some of the invariant factors is not factorable by itself because $A(s)$ has a zero on $\operatorname{Re} s=0$ and, moreover, this zero is of an odd multiplicity. So the condition is almost always satisfied and hence the spectral factor generically exists.

On the other hand, the condition is not necessary. When some of the invariant factors is not factorable by itself, the whole matrix need not but can be so, all the same:

Example 1 If some of the invariant factors is not factorable by itself, the matrix is not likely to be factorable. So is, for example the mat.rix

$$
A(s)=\left[\begin{array}{cc}
1+s^{2} & 0 \\
0 & 1+s^{2}
\end{array}\right]
$$

which is already in its Smith form. Despite the fact that its determinant (det $A(\mathbf{s})=\left(1+\mathbf{s}^{2}\right)^{2}$ ) is factorable, the matrix $A(\mathrm{~s})$ itself is evidently not.

Example 2 However, there is still a small gap and the condition is not necessary, indeed: Just consider

$$
A(\mathbf{s})=\left[\begin{array}{cc}
0 & \mathbf{s} \\
-\mathbf{s} & \mathbf{s}^{2}
\end{array}\right]
$$

Here $i_{1}(A)=\mathrm{s}$ violates the condition yet $A(\mathrm{~s})$ possesses the factor

$$
C(s)=\left[\begin{array}{cc}
1 & 0 \\
-1 & \mathbf{s}
\end{array}\right]
$$

all the same. ludeed.

$$
\left[\begin{array}{ll}
1 & -1 \\
0 & -s
\end{array}\right]\left[\begin{array}{rr}
1 & 0 \\
0 & -1
\end{array}\right]\left[\begin{array}{rr}
1 & 0 \\
-1 & s
\end{array}\right]=\left[\begin{array}{rr}
0 & s \\
-s & s^{2}
\end{array}\right]
$$

Notice that

$$
\mathcal{S}(A)=\left[\begin{array}{ll}
\mathrm{s} & 0 \\
0 & \mathrm{~s}
\end{array}\right]
$$

but

$$
\mathcal{S}(C)=\left[\begin{array}{ll}
1 & 0 \\
0 & \mathbf{s}
\end{array}\right]
$$

so that here

$$
\mathcal{S}(A) \neq \mathcal{S}\left(C^{*}\right) \mathcal{S}(C) \text {. }
$$

In fact. this situation is not quite clear as yet.

If the Hurwity spectral factor does exist, it is not unique at all. The class of solutious can be parameterized as follows:

Theorem 2 Let a polynomial matrix $C(s)$ be a Hurwitz spectral factor of $A(\mathrm{~s})$. Then also $B(\mathrm{~s})$

$$
B(\mathbf{s})=T(\mathbf{s}) C(\mathbf{s})
$$

is a Hurwilz spectral factor of $A(s)$ for any unimodular matrix $T$ (s) wuch that

$$
T^{*}(\mathbf{s}) K^{\prime} T(\mathbf{s})=K
$$

(llee so called $K$-unitary matrix)

"Due to the para-Hermitian uature of $A(5)$, all the invariant factors are "symutric": in $(A)= \pm i,(A)$.
There is, of course, a couple of substantial differences between the standard and the $J$-spectral factorization: For example, all $K$ unitary matrices appear to be of degree 0 (i.e. real $K$-orthogonal matrices) whenever $K$ is positive definite. Besides, they are sufficient to provide a particular form of $C(\mathrm{~s})$ (having one of the coefficients triangular). In contrast, for an indefinite $K$, there (xist really polyuomial $K$-unitary matrices. Moreover, they need not be able to put. ('(s) in a particular form.

Example $3 \mathrm{~A}$ s a counter-example, consider the symmetric constant matrix

$$
\left[\begin{array}{ll}
0 & 2 \\
2 & 0
\end{array}\right]
$$

Which doos not possess any triangular factorization (1). In fact, onc call casy prove lhal every factor of this matrix must have all its colries non-zero such as, e.g..

$$
\left[\begin{array}{ll}
0 & 2 \\
2 & 0
\end{array}\right]=\left[\begin{array}{rr}
1 & -1 \\
1 & 1
\end{array}\right]\left[\begin{array}{rr}
1 & 0 \\
0 & -1
\end{array}\right]\left[\begin{array}{rr}
1 & 1 \\
-1 & 1
\end{array}\right]
$$

Of course, nore frectom exists whenever $A(\mathrm{~s})$ is a singular matrix. What we have decided here is to put the whole singularity into $K$ aud led $("(s)$ be alwavs non-singular. But this is a matter of delinition.

\section{Symmetric factor extraction}

The first algorithm presented is a generalization of the procedure developed ly ('allies [1] for standard spectral factorization. The mellool refuires the following assumptions:

\section{Assumptions 3}

(3.A) $A(s)$ is nonzero on the imaginary axis.

(3.B) $A(s)$ is diagonally reduced $[1]$.

Assumption (3.A) implies the existence conditions of Theorem 1 . Assumption (3.B) causes no loss of generality because any paraHermitian matrix $A(s)$ that is not diagonally reduced can easily br made diagonally reduced [1].

The lirst step of the algorithm is to compute the zeros of $A(\mathrm{~s})$ - that is. the roots of the polynomial det $A(s)$. Because $A(\zeta)$ is para-llormitian. if $\zeta$ is a zero, so is $-\zeta$. Likewise, because $A(\zeta)$ thas real coeflicients, if $\zeta$ is a zero, so is its complex conjugate $\zeta$. Numicrically. the zeros of $A(s)$ may be obtained as the eigenvalues of the (generalized) companion matrix ${ }^{6}$ defined by the coefficient matrices of $A(s)$.

The second part of the algorithm is to extract from $A(s)$, sequentially: first-or second-order elementary factors corresponding to cach of the zoros. If $T(s)$ is such an elementary factor, we write

$$
A=T^{*} A^{\prime \prime} T
$$

where also $A^{\prime \prime}$ is polynomial. Depending on whether $\zeta$ is real or complex we distinguish three different cases for the elementary lactor $T$.

1. (anr 1. If the zero $\zeta$ is ral the elementary factor is of the

'( 'ompare K. D. (iregson and N. J. Young, "Finite representations of block Ilanked oprerators and balanced realization." Operator Theory: Adranres and Applications. Vol. 35. pp. 441-480 (1988). 
form

$T(\mathbf{s})=\left[\begin{array}{ccccccccccc}1 & 0 & \cdots & 0 & 0 & -\alpha_{1} & 0 & 0 & \cdots & 0 & 0 \\ 0 & 1 & \cdots & 0 & 0 & -\alpha_{2} & 0 & 0 & \cdots & 0 & 0 \\ \cdots & \cdots & \cdots & \cdots & \cdots & \cdots & \cdots & \cdots & \cdots & \cdots & \cdots \\ 0 & 0 & \cdots & 0 & 1 & -\alpha_{k-1} & 0 & 0 & \cdots & 0 & 0 \\ 0 & 0 & \cdots & 0 & 0 & s-\zeta & 0 & 0 & \cdots & 0 & 0 \\ 0 & 0 & \cdots & 0 & 0 & -\alpha_{k+1} & 1 & 0 & \cdots & 0 & 0 \\ \cdots & \cdots & \cdots & \cdots & \cdots & \cdots & \cdots & \cdots & \cdots & \cdots & \cdots \\ 0 & 0 & \cdots & 0 & 0 & -\alpha_{n} & 0 & 0 & \cdots & 0 & 1\end{array}\right]$

with $\alpha_{1}, a_{2}, \cdots, \alpha_{n}$ real constants to be determined. The nonunit column of $T$ is the $k$ th column, with $k$ to be determined. The factor $T$ may be seen as a variant of the Hermite standard form of a polynomial matrix factor of degree 1 . It has a single real zero $\zeta$. In more compact form we write $T$ as

$$
T(\mathrm{~s})=\left[\begin{array}{ccc}
I & -a_{1} & 0 \\
0 & \mathrm{~s}-\zeta & 0 \\
0 & -a_{2} & I
\end{array}\right]
$$

The constant vectors $a_{1}$ and $a_{2}$ may be found as follows. If $\zeta$ is a zero of $A$, clearly $A(\zeta)$ is singular, so that there exists a real constant. null vector $\epsilon$ corresponding to $\zeta$ such that

$$
A(\zeta) \epsilon=0 .
$$

Because $T^{*}(\zeta) A^{\prime \prime}(\zeta) T(\zeta) \epsilon=0$ and $\zeta$ is a zero of $T$, we may determine $a_{1}$ and $a_{2}$ by letting $T(\zeta) \epsilon=0$. Writing out this identity component-by-component, it is easily found that $a_{1}$ and $a_{2}$ follow from

$$
a=\epsilon / e_{k},
$$

where $c_{k}$ is the $k$ th component of $\epsilon$, and the constant vector $a$ is defined by

$$
a=\left[\begin{array}{c}
a_{1} \\
1 \\
a_{2}
\end{array}\right] .
$$

It remains to determine the "remaining factor" $A^{\prime \prime}$. To this end. we first ext.ract the factor $T$ "on the right" and write

$$
A=A^{\prime} T \text {. }
$$

with the square polynomial matrix $A^{\prime}$ to be determined. Multiplying the equality $A=A^{\prime} T$ out element-by-element it is easy to see that all entries of $A$ and $A^{\prime}$ are equal except those in their $k$ th columns. Denoting the $k$ th column of $A^{\prime}$ as $p_{k}^{\prime}$ it follows that

$$
A(\mathrm{~s}) a=p_{k}^{\prime}(\mathrm{s})(\mathrm{s}-\zeta)
$$

From this, $p_{k}^{\prime}$ may easily be computed. The polynomial matrix $A^{\prime \prime}$ now may be obtained by the left extraction

$$
A^{\prime}=T^{*} A^{\prime \prime},
$$

which we rewrite as the right extraction

$$
\left(A^{\prime}\right)^{*}=\left(A^{\prime \prime}\right)^{*} T
$$

This extraction follows by the same procedure as before. Because $A^{\prime \prime}$ is para-Hermitian, it is sufficient to compute the kth diagonal entry $p_{k k}^{\prime \prime}$ of $A^{\prime \prime}$. It may be solved from the equation

$$
p_{k}^{\prime *}(\mathbf{s}) a=p_{k k}^{\prime \prime}(\mathbf{s})(s-\zeta)
$$

The nondiagonal elements of the $k$ th column of $A^{\prime \prime}$ equal the corresponding entries of $p_{k}^{\prime}$, the nondiagonal elements of the $k$ th th row of $A^{\prime \prime}$ follow by adjugation, while the remaining clemcuts of $A^{\prime \prime}$ equal the corresponding elements of $A$. This define's $A^{\prime \prime}$.

2. (asc ?. If the zero $\zeta$ is complex there are two possibilities for the elementary factor $T$.

(a) Case ar. The first possibility is that $T$ has a single nonunit $k$ th column of degree two, so that $T$ is of the form

$$
T(\mathrm{~s})=\left[\begin{array}{ccc}
I & -a_{1}-b_{1} \mathrm{~s} & 0 \\
0 & (\mathrm{~s}-\zeta)(\mathrm{s}-\bar{\zeta}) & 0 \\
0 & -a_{2}-b_{2} \mathrm{~s} & I
\end{array}\right],
$$

with $a_{1}, a_{2}, b_{1}$ and $b_{2}$ real constant coefficient vectors to be determined. Extraction of this factor takes care of both the zero $\zeta$ and its complex conjugate $\bar{\zeta}$. Extraction on the right in the form

$$
A=A^{\prime} T
$$

results in a matrix $A^{\prime}$ whose elements equal those of $A$ excopt. Hose in the kth column. Writing the complex zero $\zeta$ and the corresponding (complex) null vector $e$ in Cartesian form as

$$
\zeta=\sigma+j \omega, \quad \epsilon=p+j q,
$$

denotiug the kith column of $A^{\prime}$ as $p_{k}^{\prime}$, and defining the iwo constant vectors $a$ and $b$ by

$$
a=\left[\begin{array}{c}
a_{1} \\
1 \\
a_{2}
\end{array}\right], \quad b=\left[\begin{array}{c}
b_{1} \\
0 \\
b_{2}
\end{array}\right],
$$

it may be found that

$$
\left[\begin{array}{ll}
a & b
\end{array}\right]\left[\begin{array}{cc}
p_{k} & q_{k} \\
\sigma p_{k}-\omega q_{k} & \sigma q_{k}+\omega p_{k}
\end{array}\right]=\left[\begin{array}{ll}
p & q
\end{array}\right]
$$

which may be solved for $a$ and $b$. The $k$ th column $p_{k}^{\prime}$ of $A^{\prime}$ may be obtained from the equality

$$
A(\mathrm{~s})(a+b \mathrm{~s})=p_{k}^{\prime}(\mathrm{s})(\mathrm{s}-\zeta)(\mathrm{s}-\bar{\zeta}) .
$$

The nondiagonal elenents of the $k$ th column of the remaining factor $A^{\prime \prime}$ in the extraction $A=T^{* *} A^{\prime \prime} T$ equal the corresponding elements of $p_{k}^{\prime}$, while the nondiagonal elements of the $k$ th row of $A^{\prime \prime}$ follow by adjugation. The $k$ th diagonal element $p_{k k}^{\prime \prime}$ of $A^{\prime \prime}$ may be found from the equality

$$
p_{k}^{\prime *}(\mathrm{~s})(a+b \mathrm{~s})=p_{k k}^{\prime \prime}(\mathrm{s})(\mathrm{s}-\zeta)(\mathrm{s}-\bar{\zeta}) .
$$

(b) ('ass 29. The second situation that may arise when the \%ro $\zeta$ is complex is that the elementary factor $T$ lias two nonunit columus (columns $k$ and $l$ ) of degree onc. so that it has the form

$$
T(s)=\left[\begin{array}{ccccc}
I & -a_{1} & 0 & -b_{1} & 0 \\
0 & s-\alpha & 0 & -\beta & 0 \\
0 & -a_{2} & I & -b_{2} & 0 \\
0 & -\gamma & 0 & s-\delta & 0 \\
0 & -a_{3} & 0 & -b_{3} & I
\end{array}\right],
$$

with $a_{1}, a_{2}, a_{3}, b_{1}, b_{2}$, and $b_{3}$ real constant vectors to be determined, and $\alpha, \beta, \gamma$, and $\delta$ real constants 1.) be found. Extraction on the right in the form $A$ $=A^{\prime} T$ now results in a polynomial matrix $A^{\prime}$ whose 
elements equal the corresponding elements of $A$ except those in the $k$ th and $l$ th columns. Writing the complex zero $\zeta$ and the corresponding complex null vector $e$ in Cartesian form as

$$
\zeta=\sigma+j \omega, \quad e=p+j q,
$$

and denoting the two constant vectors $a$ and $b$ by

$$
a=\left[\begin{array}{c}
a_{1} \\
1 \\
a_{2} \\
0 \\
a_{3}
\end{array}\right], \quad b=\left[\begin{array}{c}
b_{1} \\
0 \\
b_{2} \\
1 \\
b_{3}
\end{array}\right],
$$

it. may be found that

$$
\left[\begin{array}{ll}
a & b
\end{array}\right]\left[\begin{array}{cc}
p_{k} & q_{k} \\
p_{l} & q_{l}
\end{array}\right]=\left[\begin{array}{ll}
p & q
\end{array}\right],
$$

with $p_{k}$ and $p_{l}$ the $k$ th and $l$ th elements of $p$, and a similar notation for $q$. This expression allows solving for $a$ and $b$. The constants $\alpha, \beta, \gamma$, and $\delta$ follow from the identity

$$
\left[\begin{array}{ll}
\alpha & \beta \\
\gamma & \delta
\end{array}\right]=\sigma I-\omega\left[\begin{array}{ll}
p_{k} & q_{k} \\
p_{l} & q_{l}
\end{array}\right]\left[\begin{array}{rr}
0 & -1 \\
1 & 0
\end{array}\right]\left[\begin{array}{ll}
p_{k} & q_{k} \\
p_{l} & q_{l}
\end{array}\right]^{-1},
$$

with $I$ the $2 \times 2$ unit matrix. In the right extraction $A=A^{\prime} T$, the elements of $A^{\prime}$ equal the corresponding elements of $A$ except those of the $k$ th and $l$ th columns. The $k$ th and lth columns $p_{k}^{\prime}$ and $p_{l}^{\prime}$ of $A^{\prime}$ may be solved from the identity

$$
A(s)\left[\begin{array}{ll}
a & b
\end{array}\right]=\left[\begin{array}{ll}
p_{k}^{\prime}(s) & \left.q_{k}^{\prime}(s)\right]
\end{array}\left[\begin{array}{cc}
s-\alpha & -\beta \\
-\gamma & s-\delta
\end{array}\right] .\right.
$$

The elements of the factor $A^{\prime \prime}$ in the extraction $A=$ $T^{*} A^{\prime \prime} T$ equal the elements of $A$, except all elements of the $k$ th and $l$ th columns and rows. The elements of the $k$ th column of $A^{\prime \prime}$, except those in the $k$ th and $l$ th positions, equal the corresponding elements of $p_{k}^{\prime}$. Similarly, the elements of the lth column of $A^{\prime \prime}$, except those in the $k$ th and $l$ th positions, equal the corresponding elements of $p_{l}^{\prime}$. The elements of the $k$ th and $/$ th rows of $A^{\prime \prime}$, except those in the $k$ th and $l$ th positions. follow by adjugation. The elements $p_{k k}^{\prime \prime}, p_{k l}^{\prime \prime}$, $p_{l k}^{\prime \prime}$. and $p_{l l}^{\prime \prime}$ of $A^{\prime \prime}$, finally, may be obtained from the identity

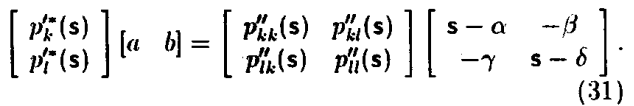

The following rules determine which case applies and which colunu $k$ or columus $k$ and $l$ are to be selected. Let $\delta_{j}, j=1,2, \cdots$, be lle degrere of the jth diagonal entry of $A$. Next, given the null vector $:$ corresponding to the root $\zeta$, define the active index set $\mathcal{A}$ as

$$
\mathcal{A}=\left\{i: \epsilon_{i} \neq 0\right\}
$$

and the highost active inder set $\mathcal{M} \subseteq \mathcal{A}$ as

$$
\mathcal{M}=\left\{i \in \mathcal{A}: \delta_{i} \geq \delta_{j} \text { for } j \in \mathcal{A}\right\}
$$

with $c$; the ith element of $e$.

I. If the zoro $\zeta$ is real, case I applies. The column number $k$ : may be any element of $\mathcal{M}$. For numerical reasons it is best to select $k$ such that $] e_{k}$ ] maximizes $e_{j}, j \in \mathcal{M}$.
2. If $\zeta$ is complex. case 21 or case 22 applies. Write $\zeta$ and $e$ in ('artesian form as

$$
\zeta=\sigma+j \omega, \quad e=p+j q .
$$

Define the matrix $E$ as

$$
E=\left[\begin{array}{ll}
p_{\mathcal{M}} & q_{\mathcal{M}}
\end{array}\right],
$$

where $p_{M}$ is the column vector whose entries consist of those elements $p_{i}$ of $p$ such that $i \in \mathcal{M}$, and $q_{\mathcal{M}}$ is similarly defined. Then if rank $E=1$, case 21 applies, while if rank $E$ $=2$, cass 22 is applicable.

(a) In case $21, k$ is any element of $\mathcal{M}$. Again, for nuunerical reasons it is best to choose $k$ such that $] e_{k}$ ] maximizes $\left.] \epsilon_{j}\right], j \in \mathcal{M}$.

(b) In case $22, k$ and $l$ are any two different elements of $\mathcal{H}$. For numerical reasons it is best to choose $k$ and $l$ such that the condition number of

$$
\left[\begin{array}{cc}
p_{k} & q_{k} \\
p_{l} & q_{l}
\end{array}\right]
$$

is maximal.

For spectral ractorization. the zero $\zeta$ of each factor that is extracted on the right is chosen to have negative real part. Then the corresponding factor that is extracted on the left has $-\zeta$ as its zero. Thic extraction procedure is repeated until the supply of zeros is exhausted. The order in which factors corresponding to the successive zeros are extracted is not important. Eventually, $A$ is reduced to the form

$$
A=\left(T^{(m)} T^{(m-1)} \ldots T^{(1)}\right)^{*} A^{(0)}\left(T^{(m)} T^{(m-1)} \ldots T^{(1)}\right),
$$

with $T^{(1)}, T^{(2)}, \cdots, T^{(m)}$ the elementary factors that successively have becn extracted.

For standard spectral factorization (where $A$ is positive definite on the imaginary axis and $J=I$ ), after each extraction the remaining factor is diagonally reduced, and the final factor $A^{(0)}$ is a symmetric real constant matrix. $A^{(0)}$, in its turn, may be factored as

$$
A^{(0)}=T^{(0)}{ }^{\mathrm{T}} r T^{(0)}
$$

by successively using each diagonal element of $A^{(0)}$ to clear the corresponding row and column, and normalizing this diagonal element to \pm 1 depending on its sign. For ordinary spectral factorization this amounts to Cholesky factorization (with $K=I$ ).

If $A$ is not positive-definite on the imaginary axis, generically $A^{(0)}$ is a simmetric constant matrix that may be factored as $A^{(0)}=T^{(0)^{\mathrm{T}}} K^{-} T^{(0)}$. After suitable permutation of the columns and rows of $K$ one obtains $K=J$. Nongenerically $A^{(0)}$ is a nonconstant unimodular para-Hermitian polynomial matrix, which may br $J$-factored as explained for the second algorithm discussed in this paper. If this situation arises, we call the $J$ spectral factorization singular. As the example below illustrates, J-spectral lactorizations that are "near-singular" lead to spectral factors will wery large coefficients.

Example 4 I3y way of example we consider the $J$-spectral factorization of the polynomial matrix ${ }^{7}$

$$
A s)=\left[\begin{array}{cc}
\left(1-\frac{2}{\lambda^{2}}\right)-2\left(1-\frac{1}{\lambda^{2}}\right) s^{2} & -1+\left(-1+\frac{2}{\lambda^{3}}\right) s \sqrt{2} \\
-1-\left(-1+\frac{2}{\lambda^{3}}\right) s \sqrt{2} & \left(1-2 \frac{c^{2}}{\lambda^{3}}\right)+\frac{2}{\lambda^{2}} s^{2}
\end{array}\right],
$$

\footnotetext{
"Taken from Section 1.12 of [i]].
} 
with $\lambda$ a real constant. Since

$$
\operatorname{det} A(\mathrm{~s})=\frac{4}{\lambda^{2}}\left(1-\frac{1}{\lambda^{2}}\right)\left(1+\mathrm{s}^{4}\right)
$$

the roots of $A$ are $\frac{1}{2} \sqrt{2}( \pm 1 \pm j)$. We assume that $\left.] \lambda\right]>1$ so that $A$ is nonsingular. Selecting the root $\zeta=\frac{1}{2} \sqrt{2}(-1+j)$, we find the corresponding null vector

$$
\epsilon=\left[\begin{array}{c}
2+j\left(-2+\lambda^{2}\right) \\
\left(-2+\lambda^{2}\right)+j\left(-2+2 \lambda^{2}\right)
\end{array}\right] .
$$

The active and highest active index set for $] \lambda]>1$ are both given by $\mathcal{A}=\mathcal{M}=\{1,2\}$. Writing $\epsilon$ in Cartesian form as $\epsilon=p+j q$ it is casily found that the determinant of the matrix

$$
E=\left[\begin{array}{ll}
p_{\mathcal{M}} & q \mathcal{M}
\end{array}\right]=\left[\begin{array}{ll}
p & q
\end{array}\right]=\left[\begin{array}{cc}
2 & -2+\lambda^{2} \\
-2+\lambda^{2} & -2+2 \lambda^{2}
\end{array}\right]
$$

is $\Delta_{\lambda}=-8+8 \lambda^{2}-\lambda^{4}$. The roots of the determinant are $\pm \sqrt{4 \pm 2 \sqrt{2}}$. For $\lambda$ not equal to one of these four values the determinant is nonzero, so that the extraction corresponding to the root $\zeta$ is of type 22 .

We select the column numbers $k$ and $l$ as $k=1, l=2$. Going through the necessary computational steps (where the assistance of Mathomatica is very helpful) the elementary factor $T_{\lambda}$ of type 22 and the "remaining factor" $A_{\lambda}^{\prime \prime}$ defined by $A=T_{\lambda}^{*} A_{\lambda}^{\prime \prime} T_{\lambda}$ may be found to be given by

$$
\begin{gathered}
T_{\lambda}(s)=\left[\begin{array}{cc}
s-\frac{8-4 \lambda^{2}-\lambda^{4}}{\Delta_{\lambda} \sqrt{2}} & -\frac{8-4 \lambda^{2}+\lambda^{4}}{\Delta_{\lambda} \sqrt{2}} \\
-\frac{-8+12 \lambda^{2}-5 \lambda^{4}}{\Delta_{\lambda} \sqrt{2}} & s-\frac{8-12 \lambda^{2}+3 \lambda^{4}}{\Delta_{\lambda} \sqrt{2}}
\end{array}\right] . \\
A_{\lambda}^{\prime \prime}(s)=\left[\begin{array}{cc}
2-\frac{2}{\lambda^{2}} & 0 \\
0 & -\frac{2}{\lambda^{2}}
\end{array}\right] .
\end{gathered}
$$

From this it easily follows that the desired $J$-spectral factorization is $A_{\lambda}=C_{\lambda}^{*} J C_{\lambda}$. with

$$
\begin{gathered}
J=\left[\begin{array}{rr}
1 & 0 \\
0 & -1
\end{array}\right], \\
C_{\lambda}(s)=\left[\begin{array}{cc}
\left(s-\frac{8-4 \lambda^{2}-\lambda^{4}}{\Delta_{\lambda} \sqrt{2}}\right) \sqrt{2-\frac{2}{\lambda^{2}}} & -\frac{8-4 \lambda^{2}+\lambda^{4}}{\Delta_{\lambda} \sqrt{2}} \sqrt{2-\frac{2}{\lambda^{2}}} \\
-\frac{-8+12 \lambda^{2}-5 . \lambda^{4}}{\Delta_{\lambda} \sqrt{2}} \sqrt{\frac{2}{\lambda^{2}}} & -\left(s-\frac{8-12 \lambda^{2}+3 \lambda^{4}}{\Delta_{\lambda} \sqrt{2}}\right) \sqrt{\frac{2}{\lambda^{2}}}
\end{array}\right] .
\end{gathered}
$$

Inspection shows that as $\lambda$ approaches one of the roots of $\Delta_{\lambda}=0$ most of the coefficients of the spectral factor $C_{\lambda}$ approach infinity.

Next assume that $\lambda$ equals one of the roots of $\Delta_{\lambda}=0$, for in stance, $\lambda=\sqrt{4+2 \sqrt{2}}$. For this value of $\lambda$ the factorization is singular. Again taking $\zeta=\frac{1}{2} \sqrt{2}(-1+j)$, the rank of the matrix $E=\left[\begin{array}{ll}p & q\end{array}\right]$ now is one, so that the extraction is of the type 21 . We choose $k=1$. With or without Mathematica it is straightforward to find that

$$
\left[\begin{array}{ll}
a & b
\end{array}\right]=\left[\begin{array}{cc}
1 & 0 \\
1+\sqrt{2} & 0
\end{array}\right]
$$

so that the elementary factor of type 21 we extract is

$$
T_{0}(s)=\left[\begin{array}{cc}
1+s \sqrt{2}+s^{2} & 0 \\
-1+\sqrt{2} & 1
\end{array}\right] \text {. }
$$

The "remaining factor" $A_{o}^{\prime \prime}=\left(T_{o}^{*}\right)^{-1} A T_{o}^{-1}$ may be found to be given by

$$
A_{i \prime}^{\prime \prime}(\mathrm{s})=\left[\begin{array}{cc}
0 & \frac{1}{2} \sqrt{2} \\
\frac{1}{2} \sqrt{2} & \frac{1+\sqrt{2}+s^{2}}{2+\sqrt{2}}
\end{array}\right] \text {. }
$$

This factor is nonconstant unimodular, which confirms that the factorization is singular. Using the unimodular factorization

$$
\left[\begin{array}{ll}
0 & a \\
0 & p
\end{array}\right]=U_{o}^{*}\left[\begin{array}{rr}
1 & 0 \\
0 & -1
\end{array}\right] U_{o}, \quad U_{o}=\sqrt{\frac{\alpha}{2}}\left[\begin{array}{ll}
1 & \frac{p}{2 \alpha}+1 \\
1 & \frac{p}{2 \alpha}-1
\end{array}\right]
$$

witl o any positive number and $p$ any symmetric scalar real polyomial, it may lee found that the singular factorization we are looking lor is $A=C_{0}^{*} J C_{0}$, where numerically

$\because=\left[\begin{array}{rr}-1.55865+0.810896 s+0.297302 s^{2} & 0.891905+0.123146 s^{2} \\ 1.3123 .5+0.8 .10896 s+0.297302 s^{2} & -0.297302+0.123146 s^{2}\end{array}\right]$.

Note that the column degrees of this factor are both two, instead of one as for the nonsingular case.

\section{Diagonalization}

The scrond algorithm to be presented here is based on the diagonalization ${ }^{8}$ of given matrix. It avoids the need to compute the zeros but it consists of nore involved polynomial operations. It works under the assumption:

Assumption (6) l.c for enery nonzero invariant polynomial $i_{1}(A)(s)$ of $A(\mathbf{s}) i=1 \ldots . . n$. holds that if it possesses zeros on $\operatorname{Re} s=0$. Hirn they must be of even multiplicity.

which clearly cotuals the condition of Theorem 1. In fact, the algoritlun provides a constructive proof of Theorem 1 as well.

Algorithm 1 For a given $A(\mathrm{~s})$ satisfying (4.A) we calculate the drisird $C(s)$ as well as the corresponding $K$ in the following steps:

STEP 1. Find a diayonal form $D(\mathbf{s})=\operatorname{diag}\left\{d_{l}(\mathbf{s})\right\}$ of $A(\mathrm{~s})$ and the corresponding unimodular matrices $V(\mathrm{~s})$ and $W(\mathrm{~s})$ such that

$$
A(\mathbf{s})=V(\mathbf{s}) D(\mathbf{s}) W(\mathbf{s})
$$

and

(a) $D(\mathrm{~s})$ is para-Hermitian $\left(D^{*}(\mathrm{~s})=D(\mathbf{s})\right)$

(b) $D(s)$ is nonnegative definite on $\operatorname{Re} s=0$

In addition. if some $d_{l}(\mathbf{s})$ appears to be a zero polynomial (duc to the singularily of given $A(\mathrm{~s})$ ), replace it by I while zroing the corresponding column of $V(s)$ at the same time. Such a way. $D(\mathbf{s})$ is always nonsingular while $V(\mathbf{s})$ is a yencralizad unimodular matrix ${ }^{9}$ (singular if $A(\mathbf{s})$ is so).

STYP 2. Perform scalar spectral factorizations of diagonal en(rics of $D(s)$

$$
d_{l}(\mathbf{s})=f_{l}^{*}(\mathbf{s}) f_{l}(\mathbf{s})
$$

and form a (nonsingular) Hurwitz polynomial matrix

$$
F(\mathbf{s})=\operatorname{diag}\left\{f_{i}(\mathbf{s})\right\}
$$

('trurly

$$
D(\mathbf{s})=F^{*}(\mathbf{s}) F(\mathbf{s})
$$

STtil 3. (ompule matrices

$$
X(\mathbf{s})=W^{-*}(\mathbf{s}) V^{\prime}(\mathbf{s})
$$

\footnotetext{
"II fart. this method appears to be a polynomial counterpart of the rational algoritum from [H].

"A (possibly singular) polynomial matrix is called generalized unimodular if all its invariant polynomials are just real constants (possibly zeros).
} 


$$
Y(s)=F^{-*}(\mathbf{s}) X(s) F^{*}(\mathbf{s})
$$

both of which appear to be polynomial and generalized unimodular. Moreover, $Y(s)$

is para-Hermitian $\left(Y^{*}(\mathrm{~s})=Y(\mathrm{~s})\right)$.

STEP 4. Find a nonsingular (unimodular) polynomial matrix $Z(s)$ along wilh a (possibly singular) real matrix $K$ so that

$$
Y(\mathrm{~s})=Z^{*}(\mathrm{~s}) K Z(\mathrm{~s})
$$

Result $A$ desired Hurvitz spectral factor is now

$$
C^{\prime}(\mathbf{s})=Z(\mathbf{s}) F(\mathbf{s}) W(\mathbf{s})
$$

ProOf:

See [9]

Particular steps of the algoritlim are standard as discussed in [9].

Example 5 Consider now the same $A(s)$ as in Example 4, i.e.

$$
A(\mathrm{~s})=\left[\begin{array}{rr}
-0.25-0.75 \mathrm{~s}^{2} & -\mathrm{s} \\
\mathrm{s} & 0.5+0.5 \mathrm{~s}^{2}
\end{array}\right]
$$

It.s diagonal form (52) is found to be

$$
D(s)=\left[\begin{array}{rr}
0.5 & 0 \\
0 & -0.25+\mathrm{s}^{2}-0.75 \mathrm{~s}^{4}
\end{array}\right]
$$

After two scalar spectral factorizations we get the diagonal factor (54)

$$
F(s)=\left[\begin{array}{rr}
1 & 0 \\
0 & 0.5774+1.5774 s+s^{2}
\end{array}\right]
$$

Then the matrix $X(s)$ from $(56)$ is

$$
X(s)=\left[\begin{array}{rr}
0.5+0.5 s^{2} & 0.375 s \\
-0.125 s-0.375 s^{5} & -0.75+0.6563 s^{2}-0.2813 s^{4}
\end{array}\right]
$$

while $Y(s)$ from (57)

$$
Y(s)=\left[\begin{array}{rr}
0.5+0.5 \mathrm{~s}^{2} & 0.2165 \mathrm{~s}+0.375 \mathrm{~s}^{3} \\
-0.216 .5 \mathrm{~s}-0.375 \mathrm{~s}^{3} & -0.75+0.6563 \mathrm{~s}^{2}-0.2813 \mathrm{~s}^{4}
\end{array}\right]
$$

Performing finally the unimodular factorization (58) we result in

$$
\begin{gathered}
J=\left[\begin{array}{rr}
1 & 0 \\
0 & -1
\end{array}\right] \\
Z(\mathrm{~s})=\left[\begin{array}{rr}
-2.7321 & 3.2321-2.049 \mathrm{~s} \\
-2.639-0.7071 \mathrm{~s} & 3.3461-1.1427 \mathrm{~s}-0.5303 \mathrm{~s}^{2}
\end{array}\right]
\end{gathered}
$$

so that the desired Hurwitz spectral factor (59) is

$$
C^{\prime}(s)=\left[\begin{array}{rr}
1.866-0.866 \mathrm{~s} & -2.7321 \\
1.9319 & -2.639-0.7071 \mathrm{~s}
\end{array}\right]
$$

which possesses its zeros in -1 and -0.5774 .

\section{Conclusions}

Unlike Ihe standard rase, the J-spectral factorization copes with polymomial matrices which are indefinite (rather than positive definitc) on the imaginary axis. This change is reflected in a varicty of diffcroul properties tackled in the paper concerning existence and unicueness (Sec פ) and numerical aspects (Sec. 3 and 1) of the solution.
The lwo numcrical algorithms which are proposed are the only methods of $J$-spectral factorization available so far. The first algorithm (Sec. 3), which is based on the generalized symmetric factor crtraction, features simplicity and it is easy to implement via MATLAB . Its stumbling block appears to be the need to calculate zeros (roots) of the given polynomial matrix. In presence of an internal singularity (Sec. 3), it behaves as follows: the precision drops when approaching such a situation, but once it is reaclied the algorithm provides a precise yet partial factor. Fortunately: this is exactly all we need to compute a reduced

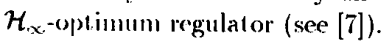

The sccond algorithm (Sec. 4), which is based on the diago"alizalion. applies for a more general class of matrices (including those witl zeros on the imaginary axis). It also obviates the need to compute zeros which is a quality one can enjoy namely in the presence of multiple zeros close to or on the imaginary axis. Finally: it handles matrices of any signature and it is believed to be numcrically altractive even in the standard (positive definite) casc. On the of her hand, it consists of more complex polynomial opcrations. III its present form, the method does not look after the internal singularity. When the given matrix

is close to it, the precision cuts down, of course, as during the first procedure but once the

phenoncron is reached, the algorithm provides precise full factor (of higher degrees).

\section{References}

[1] ('allicr l'.M.. On polynomial matrix spectral factorization by symmetric extraction, IEEE Trans. Automat. Control, AC-30, 453-464, 1985.

[2] Callier F.M. and Desoer C.A., Multivariable Feedback SysIcms. Springer Verlag, New York, 1982.

[3] Davis M.C., Factoring the spectral matrix, IEEE Trans. Automal. Control. AC:-8, 296-305, 1963.

[4] Cintmacher F.R.. The Theory of Matrices, Chelsea Publishing C'ompany, New York, 1959.

[5] Jakubovič V.A., Pactorization of symmetric matrix polynomials. Sovirt Math. Dokl., 11, 1261-1264, 1970.

[6] Kučera V.. Discrrte Linear Control: The Polynomial Approach. Wiley, ('hichester, 1979.

[i] Kiwakernaak H., The polynomial approach to $\mathcal{H}_{\infty}$-optimal regulation. In Lecturr Notes 1990 CIME Course on Recent Decelopments in $\mathcal{H}_{\mathrm{x}}$ Control Theory, Como, Villa Olmo, June 18-26. To appear, Berlin. Springer Verlag, 1991.

[8] liwakernaak H.. MATLAB macros for polynonial $\mathcal{H}_{x}$ control system optimization, Memorandum no. 881 . University of Twente, the Netherlands, 1990.

[?] S̆clock M. An algorithm for spectral factorization of polynomial malrices with any signature, Memorandum No. 91\%, Iniversity of Twente. Enschede, the Netherlands, 1990.

[10] Wiener N. and Masani L., The prediction theory of multivariate stocliastic processes, Pt. I., Acta Math., 98, 111-150, (19:5T.

[11] Youla 1).(... On the factorization of rational matrices, IRE Tintus. Inf. Theory, vol. IT-7, pp. 172-179, 1961. 